\title{
Proposta de uma Smart Classroom integrada com uma Fog Computing
}

Ivania Aline Fischer - UFSM/Santa Maria - ivaniafischer@redes.ufsm.br

Roseclea Duarte Medina- UFSM/Santa Maria - rose@inf.ufsm.br

Larissa Soute del Rio - UFSM/Santa Maria - larissa@redes.ufsm.br

Felipe Becker Nunes - AMF/Restinga Sêca - nunesfb@gmail.com

Éder Paulo Pereira - UFSM/Santa Maria - unijic@gmail.com

Resumo. A utilização da Internet das Coisas (Internet of Things - IoT) tem crescido nos mais variados contextos, entre eles o educacional. Esse artigo apresenta o emprego da IoT em uma sala de aula inteligente, que propicie um ecossistema facilitador de um processo de ensino-aprendizagem ubíquo e ciente do contexto. No desenvolvimento do ecossistema educacional observa-se que alguns problemas são acentuados, como utilização da banda de rede, tempo de resposta grande. Sendo assim, a contribuição desse trabalho é apresentar uma arquitetura que minimize esses problemas utilizando, para tanto, uma Fog adicionada a arquitetura de funcionamento da Smart Classroom. Com o intuito de validar a proposta dois cenários de aplicação são apresentados, uma Smart Classroom utilizando Fog e outra apenas utilizando Cloud. Nos dois casos é possível obter um tempo de resposta das comunicações do ambiente que utiliza Fog 20\% maior comparado com o que não utiliza. Tornando-se um ambiente com maior usabilidade dos atuadores envolvidos no processo.

Palavras Chave. Internet das coisas, Sala de aula inteligente, Computação em névoa.

\section{Proposal of a Smart Classroom composed by Fog Computing}

\begin{abstract}
The use of Internet of Things (IoT) has grown in the most varied contexts, among them the educational one. This article presents the use of IoT in an intelligent classroom, which provides an ecosystem that facilitates a ubiquitous and context-aware teaching-learning process. In the development of the educational ecosystem it is observed that some problems are accentuated, such network bandwidth utilization, large response time. Thus, the contribution of this work is to present an architecture that minimizes these problems by using a Fog added to the operating architecture of Smart Classroom. In order to validate the proposal two applications scenarios are presenteds in this work, a Smart Classroom using Fog and another only using Cloud. In both cases it is possible to obtain a response time of the communications of the environment that uses Fog 20\% greater compared to the one that does not use. Becoming an environment with greater usability of the actuators involved in the process.
\end{abstract}

Keywords. Internet of things, Smart classroom, Fog Computing.

\section{Introdução}

O uso da Internet das Coisas ao decorrer dos anos passou a ser mais intenso, acentuado pelo acréscimo de objetos inteligentes como óculos, relógios, geladeiras, fogões e carros, utilizados no dia a dia das pessoas. A partir disso, tem sido visto como precursor da 
revolução tecnológica, com o princípio de exercer um papel importante em diversas áreas como: saúde, transporte, agricultura, segurança e educação (Bath and Bath, 2017).

No cenário educacional o conceito de sala de aula inteligente (do inglês, smart classroom) bem como, de campus inteligente (do inglês, smart campus) tem o enfoque no processo de ensino e aprendizagem, objetivando torná-lo adaptativo e centrado no usuário. A aplicação e desenvolvimento da IoT integrada em uma sala de aula e/ou campus inteligente, visa aprimorar todo o processo educacional dos atores envolvidos no processo. Considerando que o modelo padrão de ensino tem sido aperfeiçoado ao passar dos anos, tornando-se mais heterogêneo, colaborativo e conectado, percebe-se claramente a necessidade de mais estudos neste novo cenário (Silva, 2017).

De acordo com Chang (2015) a utilização de IoT é um meio auxiliador na solução de problemas comuns em sistemas de ensino monolíticos, por exemplo, a fragmentação do tempo de aprendizagem e pouca dinamicidade dos materiais de ensino. Nesse âmbito, o espaço de sala de aula inteligente propicia o desenvolvimento de diversas abordagens, como controle de acesso dos alunos, experimentação remota, controle de variáveis do ambiente e demais fatores. Tais elementos oportunizam o desenvolvimento de um ambiente de ensino-aprendizagem que considera aspectos físicos, cognitivos e lógicos.

Entretanto, ao suprir as necessidades de ensino monolítico, surgem outros fatores discriminantes dentro do ambiente IoT educacional. Um deles é o grande número de dados coletados no ambiente, os quais, seguindo o princípio arquitetural da IoT, devem ser enviados à nuvem (do inglês, Cloud). Porém, esse envio além de utilizar largura de banda da rede, não atende os requisitos de latência de ambientes sensíveis IoT. Visando mitigar esta questão, pesquisas recentes têm explorado a implantação e uso da Fog Computing (traduzida como computação em névoa), (Gia, 2015) (Gupta, 2017), uma tendência que cresceu com a evolução da IoT. A Fog é vista como um método de tratamento e gerenciamento de dados nos extremos de uma rede IoT a qual objetiva o processamento e controle de um grande número de dados, antes que eles sejam enviados para a Cloud (Bonomi, 2012).

Portanto, o objetivo desse artigo é apresentar um recorte do projeto da Smart Classroom blind review, onde é proposto o uso de Fog na arquitetura geral IoT, para atender às necessidades de pré-processamento e envio de informações de aspectos físicos, cognitivos e lógicos para Cloud. Bem como, destaca-se sua real importância por meio do desenvolvimento de um caso de uso aplicado a Smart Classroom.

\section{Smart Classrooms}

Smart Classroom pode ser definida como um ambiente inteligente com consciência de contexto e gerenciamento no âmbito de aprendizagem, onde o conteúdo educacional é apresentado de forma dinâmica e eficiente, aprimorando a interação entre os alunos e o meio educacional (Huang, 2012).

A principal característica de uma Smart Classroom é permitir a manipulação dos recursos disponíveis na sala de aula automáticamente, adequando-se tanto para os alunos quanto para professores, como por exemplo, computadores, projetores, aparelhos de iluminação. Dessa forma, o ambiente inteligente caracteriza-se pela interação natural entre os seres humanos e os dispositivos eletrônicos. Um modelo sistemático das salas de aula inteligentes foi proposto por Song (2014) onde são apresentados aspectos práticos para sua implemetação, subdivididos em seis módulos: infraestrutura, rede de sensores, gerenciamento, realidade aumentada, gravação em tempo real e tecnologia ubíqua. Nesses seis módulos, tanto sistemas de softaware quanto hardware compõem o ambiente geral da sala de aula, dentre eles tem-se: o espaço fisíco da sala de aula, dispostivos finais de autenticação (RFID), sensores de batimentos cardíacos, sensores do ambiente, 
métodos de monitoramento e alerta do ambiente, uso de robôs, integração ao AVEA (Ambiente Virtual de Ensino-Aprendizagem).

De maneira geral, uma sala de aula comum quando integrada com a tecnologia IoT pode transforma-se em um ambiente que escuta e analisa vozes, movimentos, conversas, comportamento, etc. Isso possibilita uma aula mais consistente e com o alcance de impacto maior enquanto o processo de aprendizagem é menos trabalhoso, mais agradável e divertido (Glicorić, 2012). Para isso esses ambientes possuem uma série de dispositivos heterogêneos que necessitam interagir entre si, neste contexto, surge a necessidade de padronização da arquitetura para comunicação desses módulos. Para tanto, esse artigo propõe uma arquitetura com a Fog que engloba vários módulos na composição da Smart Classroom.

\section{Fog Computing}

Vista como extensão de uma Cloud para a borda da rede (Cisco, 2014), a Fog, além de auxiliar no processamento de dados, possui armazenamento disponível próximo aos sensores e atuadores para respostas rápidas das solicitações, sem a necessidade de enviar dados à Cloud para isso. Em contraste à Cloud, que tende a ser mais centralizada, uma Fog tem a visão de ser mais descentralizada (Aazam, 2014), como por exemplo, a existência de várias Fogs distribuídas por centros de pesquisa em um campus inteligente de uma universidade. Em sua essência, trabalha com dados de IoT localmente, fazendo uso de dispositivos de borda próximos aos usuários para obter armazenamento, comunicação, controle, configuração e gerenciamento do ambiente (Dastjerdi, 2016).

A disponibilidade de resposta rápida traz o benefício de baixa latência em um ambiente crítico. Torna-se como exemplo, o caso do carro inteligente que necessita baixa latência para sua execução visto que os comandos importantes de seu uso precisam ser ágeis como acionar os freios por exemplo. Com o uso da Fog no caso do carro a preocupação do atraso de resposta de comandos para o seu funcionamento é amenizado uma vez que a mesma encontra-se mais próxima da execução. Com isso, nota-se que a Fog traz benefícios em ambientes IoT como, redução de pontos de falha pois os elementos da rede são tratados de forma independente, isto motiva estudos de sua aplicabilidade.

\section{Trabalhos Relacionados}

Esta seção discute trabalhos existentes na literatura que apresentam propostas para salas de aula inteligentes com o uso de conceitos como computação pervasiva, computação em nuvem, entre outros. A sala de aula inteligente apresentada em Chang and Jung (2011) consiste no uso de cartões RFID (Radio-Frequency Identification), onde cada aluno possui seu cartão. Ao chegar na sala, o aluno passa o cartão em um quadro, que tem como função escanear o mesmo. Dessa forma, a presença do aluno em cada aula é automatizada.

Já no trabalho de Suo et al. (2009), os autores desenvolveram uma sala de aula inteligente baseada em sistemas web. Foi construída uma plataforma denominada Open Smart Classroom. Essa plataforma pode ser utilizada em diferentes localizações, já que uma das principais funções é transmitir ao vivo o professor ministrando determinada aula, além de disponibilizar a tradução da aula conforme a língua nativa dos estudantes.

A proposta do trabalho de Dekdouk (2012) é a integração de uma plataforma móvel com tecnologias pervasivas, que têm como objetivo facilitar o acesso aos conteúdos multimídia das aulas. Basicamente os estudantes e o professor possuem um tablet, sendo que, quando adentram a sala de aula os sensores detectam o código RFID de cada usuário presente no tablet e fazem o registro no servidor da plataforma. 
Automaticamente, o aluno tem acesso aos conteúdos didáticos e ao final da aula é feito o update para o servidor na Cloud, informando o progresso em relação ao conteúdo visto.

Os trabalhos citados apresentam propostas bastante diferentes, mas todas com o mesmo objetivo, automatizar as tarefas cotidianas de uma sala de aula. Em relação ao proposto neste presente trabalho, observa-se que o uso de sensores biométricos tornam o sistema de registro de frequência bem mais seguro, já que no caso do uso de cartões RFID, nada impede que um mesmo aluno coloque dois cartões no quadro.

Nenhuma das propostas apresentadas busca tratar as informações antes de elas serem enviadas para seu destino, ou seja, ao enviar uma grande quantidade de dados para a nuvem há a necessidade do uso de uma banda muito grande, acarretando em lentidão e perda de pacotes. Além disso, pode prejudicar os demais serviços que rodam na rede. Dessa forma, alguns dos principais diferenciais desta pesquisa estão centralizados no uso de sensores biométricos na identificação dos alunos, assim como a implementação de uma Fog para o tratamento dos dados transmitidos entre os dispositivos de IoT e a Cloud.

\section{Proposta da Smart Classroom}

A sala de aula inteligente blind review tem, entre vários objetivos, integrar o sistema acadêmico de uma instituição de ensino com o ambiente que compõe a sala de aula (diferentes sensores e atuadores), e o AVEAs (ambiente virtual de ensino-aprendizagem) ciente de contexto, visando proporcionar melhor flexibilidade e personificação do processo de aprendizagem. Além disso tem o enfoque na otimização de tarefas do professor, como em registro automático de presenças e de aulas, acompanhamento e reação em tempo real do processo de ensino-aprendizagem dos alunos integrantes da sala.

Para ilustrar a composição dessa Smart Classroom a Figura 1 exemplifica a arquitetura que compõe a mesma tendo o enfoque na correlação dos alunos e o ambiente.

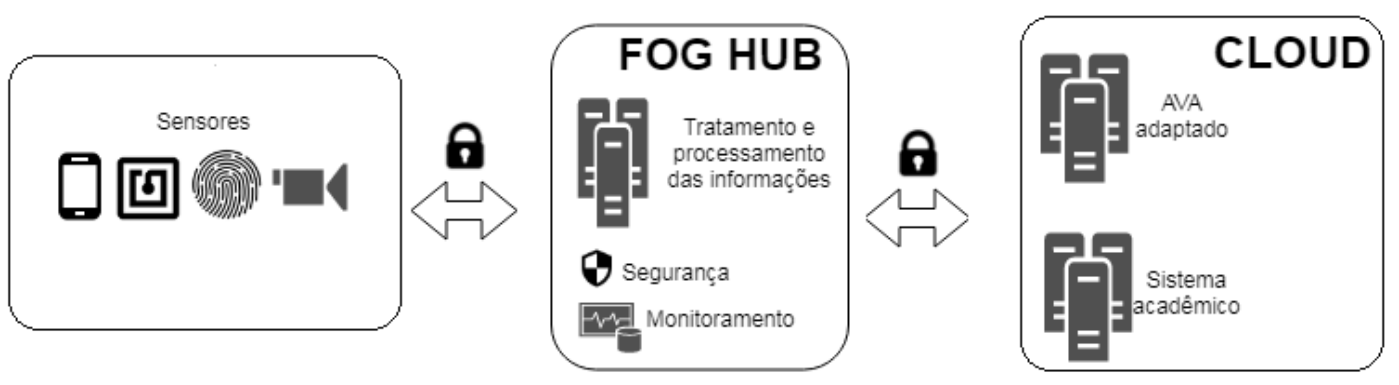

\section{Figura 1. Arquitetura da Smart Classroom}

O cenário apresentado possui uma divisão baseada em três camadas, conforme ilustrado, que permite separar níveis de tratamento dos processos ocorridos no ambiente. O processo que pode ser designado como inicial contempla os sensores da Smart Classroom, que dentre eles cita-se os smartphone, câmera, etc. Onde os mesmos irão colaborar com a automatização de rotinas burocráticas, como lançamento de aulas e marcação de presença. Suas informações são tratadas e integradas com o Ambiente Ubíquo, e são enviadas à Cloud para a integração dos sistemas acadêmicos. O objetivo da camada designada como Fog também é preocupar-se com os processos ambientais da sala de aula (temperatura, etc.).

As informações geradas das interações entre sensores e o meio gerenciador (Fog) são transferidas de forma protegida, com o uso de protocolos de segurança (SSL, Autenticação de chave assimétrica) adequados e tratadas de acordo com as premissas estabelecidas pela Fog. Por exemplo, o sensor de controle de acesso, como o biométrico, faz a leitura digital tanto do aluno quanto do professor e passa para o Fog processar as 
informações adquiridas, sendo essa a segunda camada da arquitetura. Para o processamento desses dados o uso de Fog é necessária na sala de aula inteligente, onde os dados são enviados para que ela os processe e extraia o que será encaminhado à Cloud ou mandado como resposta para algum atuador.

A ultima camada é a Cloud sendo ela integrada à um AVEA adaptado e o sistema acadêmico da instituição, que por sua vez automatiza o processo de lançamento de presença do aluno bem como o lançamento da aula no portal do professor, a partir do compartilhamento desses dados com a Fog. Em um ambiente ubíquo, os sistemas ajustam-se aos usuários e agem de forma transparente, nesse contexto o AVEA adaptado se ajustará a cada aluno considerando o nível de conhecimento, a expertise bem como seu estilo cognitivo de aprendizagem. Com a finalidade de esclarecer a composição da camada dos sensores da sala de aula inteligente a subseção 5.1 é apresentada.

\subsection{Componentes e funcionalidades}

Nessa seção são abordadas as funções dos componentes que integram a sala de aula inteligente, a fim de esclarece-los com precisão a Figura 2 é apresentada.

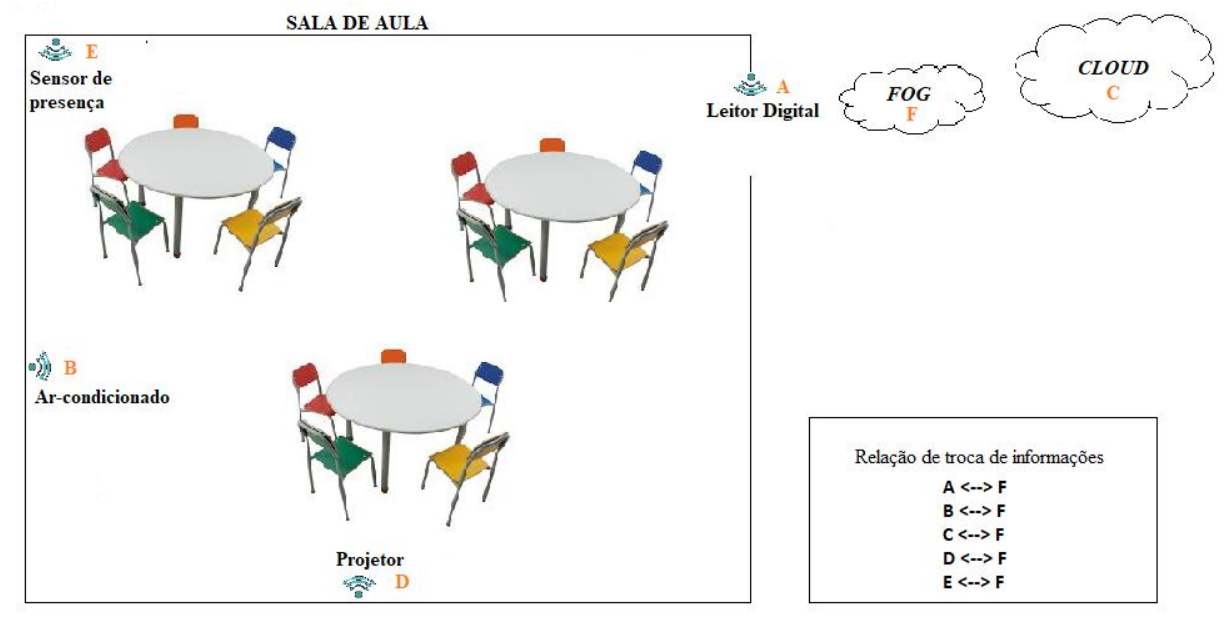

Figura 2. Componentes da sala de aula inteligente

Como pode-se notar a sala de aula é composta por quatro sensores principais indicados por letras, suas funcionalidades são destacadas a seguir:

- Controle de acesso a sala de aula (letra A): o sensoriamento dos alunos da sala de aula auxilia no processo de marcação de presença dos mesmos no sistema institucional. O componente utilizado é um sensor biométrico (FingerTech) que objetiva a criação de um ambiente ubíquo focado na aprendizagem dos indivíduos.

- Controle de temperatura e oxigênio no ar (letra B): estudos argumentam que o nível de oxigênio na sala de aula interfere na concentração do aluno (baixa pulsação cardíaca segundo Oliveira et al. (2017)), controlar a temperatura e oxigênio pode fazer diferença no processo de aprendizado. Nesse controle tem-se uma lista de temperaturas adequadas, caso a temperatura coletada pelo sensor for diferente do intervalo da lista um comando de alteração será lançado. O sensor de temperatura utilizado é o DHT11.

- Controle de presença para economia de energia: na sala de aula inteligente o sensor que controla a presença (letra $\mathrm{E}$ ), ao detectar a sala vazia, fará o desligamento/acionamento automático do ar-condicionado (letra B), das lâmpadas e projetor (letra D). Esse controle não relaciona-se diretamente ao aprendizado, mas ao uso consciente dos recursos bem como ao controle de gastos na instituição. Para isso é utilizado o sensor HC-SR501. 
- Sensor de luminosidade: o objetivo do sensor é voltado ao auxílio do professor em sala de aula. Por exemplo, quando o professor deseja ligar o projetor da sala inúmeros processos devem ser realizados. Logo, com um sensor de luminosidade apenas uma ação do professor seria necessária. A ideia é de que quando o professor desligue as lâmpadas da sala o sensor detecte a baixa luminosidade e presença na sala e assim começa o processo de ligar o projetor. $\mathrm{O}$ sensor de luminosidade utilizado é o LDR.

Para finalizer os processos de comunicação do ambiente, são esquematizadas as possíveis comunicações entre sensores e Fog e sensores e Cloud e como elas ocorrem, na seção 6 processos dessas comunicações são exemplificados.

\section{Implementação}

No decorrer do processo de implantação de um trabalho é comum deparar-se com problemas não previstos no momento de análise textual. $\mathrm{Na}$ implementação da sala de aula inteligente dissertada nesse artigo tem-se o conhecimento prévio de problemas como: aceitação social (Tomas, 2018), escalabilidade, grande utilização da largura de banda causado pelo Big Data.

A partir disso, este trabalho concentra-se no problema de Big Data, propondo o pré-processamento dos dados, localmente, antes de enviá-los à Cloud, bem como de aplicação de QoC (avaliar a qualidade da informação de contexto no ambiente), através da utilização de uma Fog. Em termos gerais a estrutura básica do processo de funcionamento da Fog da Smart Classroom é representada pela Figura 3.

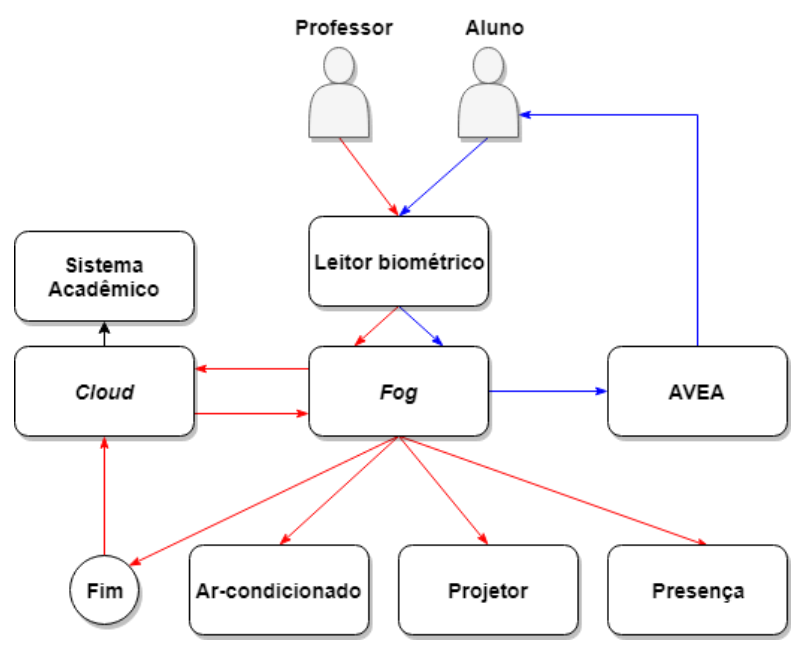

Figura 3. Processo de funcionamento da Smart Classroom

Como percebe-se na Figura 3 dois processos básicos são realizados, professor e aluno, o funcionamento da sala de aula inteligente é iniciado pela leitura da digital do professor, caso o aluno tente abrir e iniciar uma aula será gerado um erro. Isso acontece porque a relação de professores e turmas ficam alocadas em todas as Fogs das salas de aula, enquanto a relação dos alunos da turma permanecem na Cloud e são buscados pela Fog a cada nova turma.

Após a Fog constatar que é a digital de um professor ela abre a porta e aciona todos os sensores que compõem a sala de aula. A partir desse momento extrai da Cloud a relação dos alunos e mantém as informações localmente até o término da aula, após envia os dados referente aos alunos presentes para a Cloud e deleta a relação da turma. Logo, o aluno ao chegar à sala de aula é autenticado, a marcação de presença é processada pela Fog e conteúdos da aula enviados para o AVEA do aluno. Nessa estrutura, a Fog é o meio 
de comunicação entre os dispositivos da sala de aula inteligente e a Cloud que tem a missão de manter dados importantes momentaneamente e envia apenas o necessário. A seção 6.1 apresenta testes e resultados do ambiente com o uso e sem o uso de Fog.

\subsection{Ambiente de aplicação de testes}

Como nota-se, por exemplo, dados dos sensores relacionado ao ambiente não necessitam realizar comunicações com a Cloud sendo tratados localmente pela Fog. Uma aplicação real da comunicação de um sensor com o ambiente é demonstrada na Figura 4, que representa dois processos de comunicação: sensor digital (coleta os dados biométricos dos alunos para marcar presença) transmitindo dados para Fog e sensor digital transmitindo dados para Cloud.

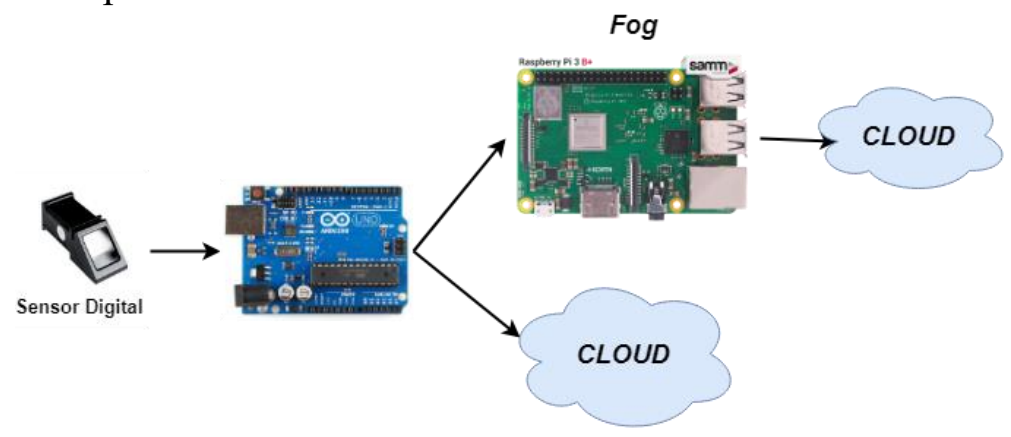

Figura 4. Método de tratamento do processo de leitura biométrica

Os testes de leitura de digitais, ar-condicionado, luzes, estão em fase avançada, assim como a implantação da Fog, que atualmente é Raspberry v3.0. Para esclarecer o processo de comunicação dos dois casos de leituras de digitais apresentados, modelos de código são demonstrados. A Figura 5 representa o modelo de coleta de dados pela Fog. Nesse processo para iniciar uma aula necessariamente a digital de um professor terá que ser lida no sensor biométrico, a Fog tem um banco local com os dados de todos os professores e horários de aula. Com essas informações a mesma solicita à Cloud a relação dos alunos que compõem aquela turma para prosseguir na validação os mesmos. $\mathrm{O}$ processo de validação até o término da aula é realizado localmente e ao finalizar a mesma as informações são atualizadas na Cloud, no sistema acadêmico institucional.

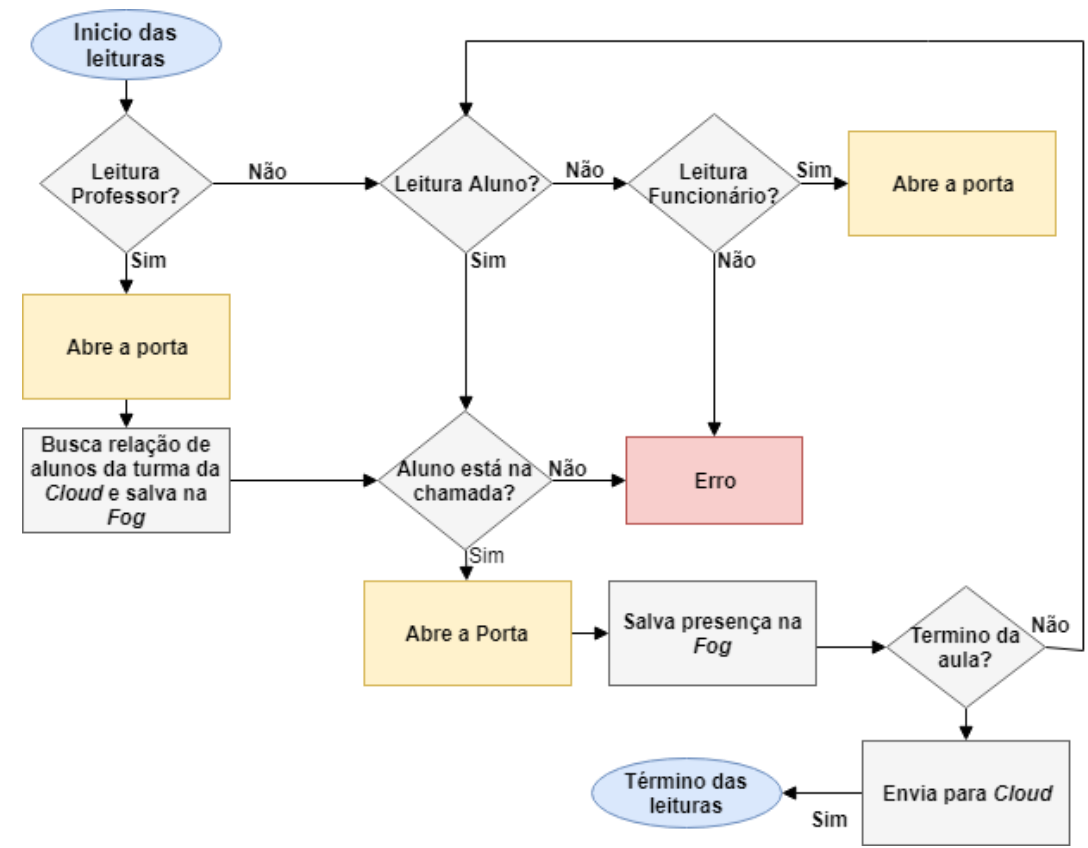

Figura 5. Fluxograma de processos utilizando Fog 
Enquanto a Figura 5 apresenta parte da implementação da Fog dentro do contexto da sala de aula inteligente, a fase abordada nesse momento é apenas dos dados do sensor biométrico que identifica os alunos. Já a Figura 6 tem o objetivo de apresentar o fluxograma de comunicação dos sensores diretamente com a Cloud.

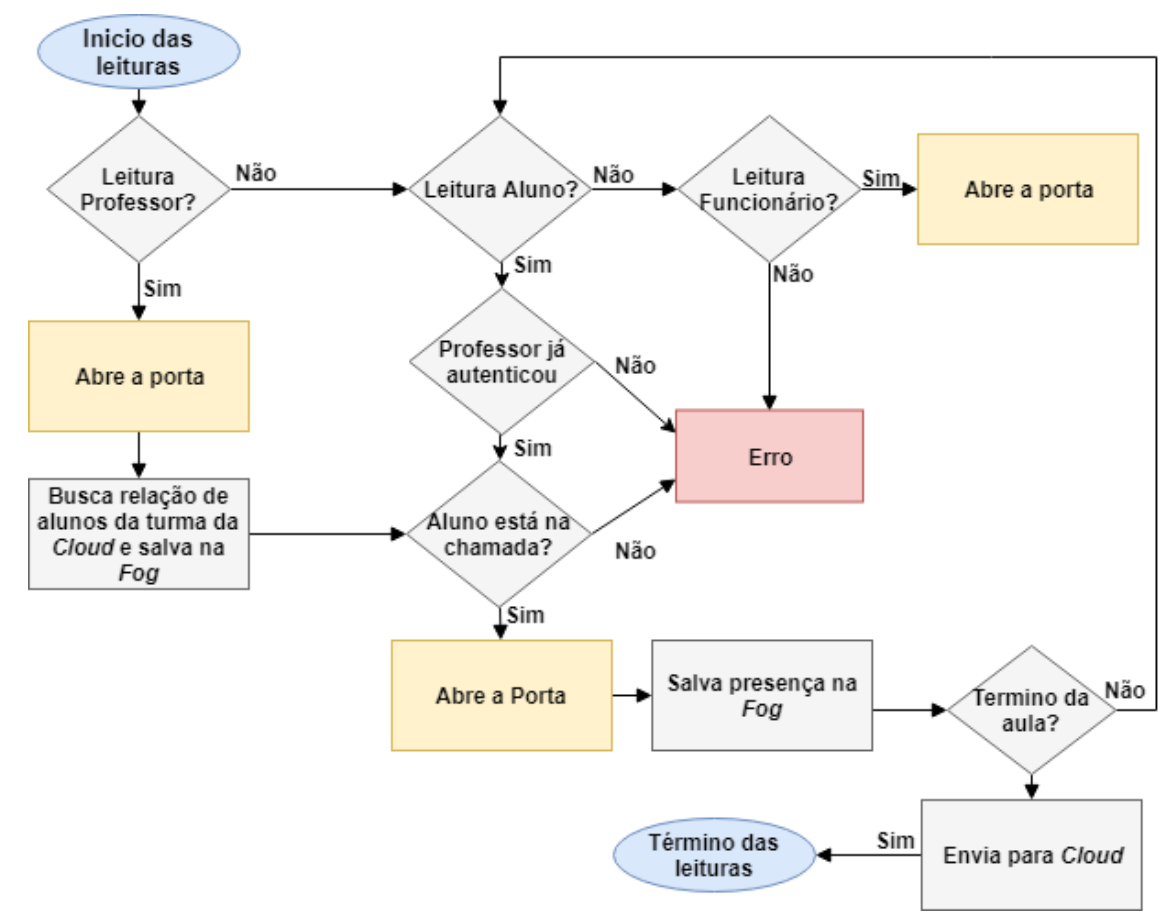

Figura 6. Fluxograma de processos apenas com Cloud

A Figura 6 representa as comunicações sem a Fog na composição do cenário, nota-se portanto que o número de processos apenas com o uso da Cloud é menor uma vez que não tem o componente intermediário de conexão. No entanto, esses processos não trazem malefícios ao tempo de transmissão das mensagens e sim benefícios uma vez que a comunicação de todas as leituras dos alunos é realizada localmente com o uso de Fog tendo assim um aspecto positivo ao ambiente. Com o intuito de comprovar o uso da Fog como método de melhoria de latência, gerenciamento e segurança a seção 7 apresenta os testes realizados.

\section{Resultados}

Para validar e justificar o uso da Fog na Smart Classroom foram realizados testes de tempo de resposta, sendo eles: tempo de resposta da comunicação do sensor biométrico à Fog e tempo de resposta do sensor biométrico à Cloud.

A ferramenta utilizada para obter os dados de tempo das transmissões foi a Wireshark (https://www.wireshark.org/), a análise do tempo de resposta é adquirida a partir da soma o tempo de ida mais o tempo de resposta da mensagem. Para aplicação de testes foi simulado a abertura de uma turma e a coleta da impressão digital de um aluno é adquirida e enviada para as Clouds chamadas HiveMQ (https://www.hivemq.com/tryout/) e Mosquitto (https://www.iot.eclipse.org/) que dispõem do broker para comunicação via MQTT (Message Queuing Telemetry Transport).

Os testes são subdivididos em totais e parciais, totais são o tempo de resposta de várias comunicações simultâneas, parciais são comunicações unicast onde a rede não está sobrecarregada. Os resultados obtidos estão representados na Tabela 1 que apresenta a resposta dos testes realizados. 
Tabela 1. Dados do tempo de resposta das conexões

\begin{tabular}{|c|l|l|l|}
\hline & Com Fog & Cloud HiveMQ & Mosquitto \\
\hline Tempo de resposta Parcial & $2,58 \mathrm{~s}$ & $13,11 \mathrm{~s}$ & $8,53 \mathrm{~s}$ \\
\hline Tempo de resposta Total & $5,96 \mathrm{~s}$ & $16,78 \mathrm{~s}$ & $35,96 \mathrm{~s}$ \\
\hline
\end{tabular}

Para evidenciar as diferenças de tempo de resposta de maneira visual a Figura 7 é apresentada, onde um gráfico é gerado para isso.

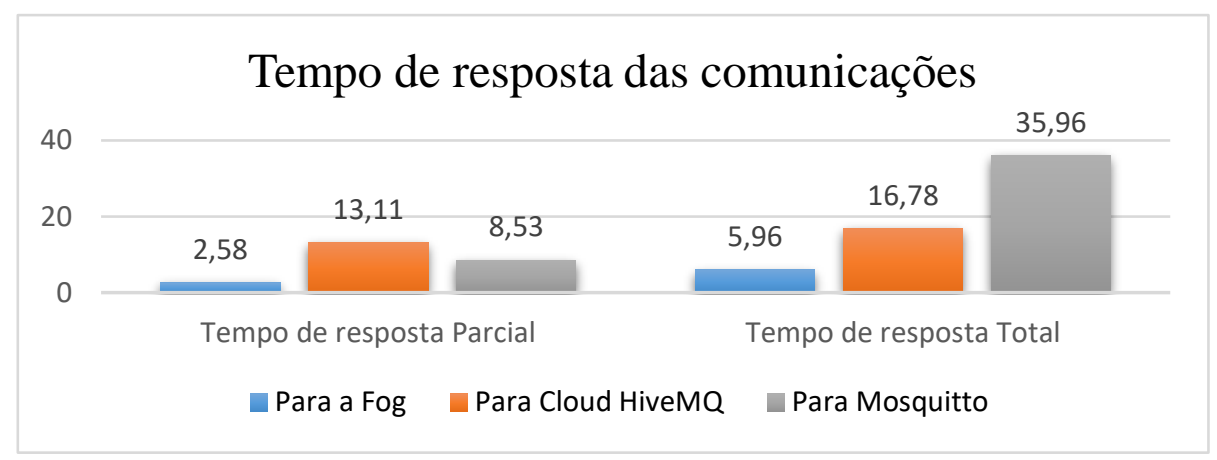

Figura 7. Tempo de resposta das comunicações da Smart classroom

Nota-se que o tempo de reposta de conexões para a Cloud tem um tempo maior, sendo esse um dos motivos para fazer uso de $F o g$, ou seja, diminuir a latência já que mesmo em uma Smart Classroom problemas desse tipo devem ser levados em consideração. Por exemplo, se a conexão de autenticação do aluno demorar um longo tempo pode gerar fila para os mesmos acessarem a sala de aula e causar transtornos.

Contudo, a Fog além de diminuir a latência objetiva a dimunuição da largura de banda da instituição que muitas vezes é precária. Essa nova abordagem na arquitetura de comunicação tem o enfoque na melhoria do processo de ensino-aprendizagem dos atores envolvidos na instituição de ensino.

\section{Conclusão}

Este artigo objetiva apresentar a proposta de desenvolvimento de uma Smart Classroom a qual é composta por inúmeros meios de auxílio no processo de ensino-aprendizagem e tem o enfoque no uso da Fog para isso. Visto que implementações da IoT não têm demostrado procupação tão acentuda em relação ao uso de $F o g$, este trabalho traz esse diferencial. Pois faz uso da tecnologia para auxiliar na diminuição do grande uso de largura de banda da Smart Classroom bem como diminui o tempo de resposta das requisições dos sensores do ambiente, consequentemente deixando o ambiente com uma melhor usabilidade.

Portanto, o artigo apresentou uma visão geral da sala de aula inteligente em desenvolvimento, a qual é focada em uso de sensores e atuadores com baixo índice de erro no reconhecimento e exatidão da identidade do aluno e a estrutura de préprocessamento que subsidiará toda essa integração. Aplicados em dois cenários do ambiente, no uso do meio intermediário de processamento e com o uso direto da Cloud.

A Smart Classroom utilizando Fog apresenta resultados parciais promissores. Os próximos passos serão a implementação de segurança e gerenciamento na Fog. Simultaneamente com a preocupação com latência e uso de banda do ambiente, pois os mesmos têm um papel importante e serão levados em consideração sempre que possível. 


\section{Referências Bibliográficas}

AAZAM, M. and HUH, E. N. (2014). Fog computing and smart gateway based communication for cloud of things. International Conference on future internet of things and cloud (ficloud), 464-470.

BHATT, J. and BHATT, A. (2017). Iot techniques to nurture education industry: Scope opportunities. International journal on emerging technologies, 128-132.

BONOMI, F., RODOLFO, M., Jian, Z., and SATEESH A. (2012). Fog computing and its role in the internet of things. ACM New York, 13-16 edition.

CHANG, C. H. and JUNG, C. C. (2011). Smart classroom roll caller system with iot architecture. IEEE Computer Society.

CHANG, C., LIN, P., TSENG, C., KONG, Y., LIEN, W., WU, M., and WU, C. (2015). Design and implementation of iot-enable mobile e-learning platform for medical education. ACM New York, 385-386 edition.

CISCO (2014). Iox overview. http://goo.gl/n2mfiw.

DASTJERDI, A. V. and RAJKUMAR, B. (2016). Fog computing: Helping the internet of things realize its potential. Computer, 112-116 edition

DEKDOUK, A. (2012). Integrating mobile and ubiquitous computing in a smart classroom to increase learning effectivenes. Conference on Education and eLearning Innovation.

GIA, T. N., JIANG, M., RAHMANI, A. M., WESTERLUND, T., LILJEBERG, P., \& TENHUNEN, H. (2015). Fog computing in healthcare internet of things: A case study on ecg feature extraction. In Computer and Information Technology (CIT), IEEE International Conference on (pp. 356-363).

GLIGORIĆ, N., UZELAC, A., \& KRCO, S. (2012). Smart classroom: real-time feedback on lecture quality. In Pervasive Computing and Communications Workshops IEEE International Conference on (pp. 391-394).

GUPTA, H., DASTJERDI, A., GHOSH, S. K., \& BUYYA, R. (2017). iFogSim: A toolkit for modeling and simulation of resource management techniques in the IoT, Edge and Fog computing environments. Software: Practice and Experience, 1275-1296.

HUANG, R., YONGBIN, H., JUNFENG, Y., and GUANGDE, X. (2012). The functions of smart classroom in smart learning age. Open Education Research.

OLIVEIRA, A. L., and Branco, M. (2017) Efeitos da Hipóxia na Variabilidade da Frequência Cardíaca em Indivíduos Saudáveis: Uma Revisão Sistemática. Int cardiovasc, v. 30, n. 3, p. f: 251-1: 261.

PIRES, P. D., BATISTA, F. T., BARROS, T., CAVALCANTE, E., and PITANGA M. (2015). Plataformas para a internet das coisas. XXXIII Simpósio Brasileiro de Redes de Computadores e Sistemas Distribuídos (SBRC).

SILVA, R. A., GABRIEL, J. N., RUBEM, C., and CALADO, I. (2017). Aplicando internet das coisas na educação: Tecnologia, cenários e projeções. Anais dos Workshops do Congresso Brasileiro de Informática na Educação. 2017. p. 1256.

SONG, S., XIAOLIU, Z., JING, D., and DUTTA S. (2014). Smart classroom: from conceptualization to construction. Conference on Intelligent Environments.

SUO, Y., NAOKI, M., HIROKI, M., and YUANCHUN, S. (2009).Open smart classroom: Extensible and scalable learning system in smart space using web service technology. IEEE transactions on knowledge and data engineering, v. 21, n. 6, p. 814-828, 2009.

TEIXEIRA, T., Hachem, S., Valérie, I., and Nikolaos, G. (2011). Service oriented middleware for the internet of things: A perspective. In: European Conference on a Service-Based Internet. Springer, Berlin, Heidelberg, 2011. p. 220-229. 